\title{
Metalloporphyrin intercalation in liposome membranes: ESR study
}

\author{
Dariusz Man $\cdot$ Rudolf Słota $\cdot$ Małgorzata A. Broda $\cdot$ \\ Giuseppe Mele $\cdot$ Jun Li
}

Received: 27 April 2010/Accepted: 4 October 2010/Published online: 21 October 2010

(C) The Author(s) 2010. This article is published with open access at Springerlink.com

\begin{abstract}
Liposomes characterized by membranes featuring diverse fluidity (liquid-crystalline and/or gel phase), prepared from egg yolk lecithin (EYL) and dipalmitoylphosphatidylcholine (DPPC), were doped with selected metalloporphyrins and the time-related structural and dynamic changes within the lipid double layer were investigated. Porphyrin complexes of $\mathrm{Mg}(\mathrm{II}), \mathrm{Mn}(\mathrm{III}), \mathrm{Fe}(\mathrm{III})$, $\mathrm{Co}(\mathrm{II}), \mathrm{Ni}(\mathrm{II}), \mathrm{Cu}(\mathrm{II}), \mathrm{Zn}$ (II), and the metal-free base were embedded into the particular liposome systems and tested for $350 \mathrm{~h}$ at $24^{\circ} \mathrm{C}$ using the electron spin resonance (ESR) spin probe technique. 5-DOXYL, 12-DOXYL, and
\end{abstract}

Electronic supplementary material The online version of this article (doi:10.1007/s00775-010-0715-1) contains supplementary material, which is available to authorized users.

\section{Man}

Institute of Physics,

Opole University,

Oleska 48,

45-052 Opole, Poland

R. Słota $(\bowtie) \cdot$ M. A. Broda

Faculty of Chemistry,

Opole University,

Oleska 48,

45-052 Opole, Poland

e-mail: rslota@uni.opole.pl

\section{G. Mele}

Dipartimento di Ingegneria dell'Innovazione,

Università del Salento, Via Arnesano,

73100 Lecce, Italy

\section{J. Li}

Key Laboratory of Synthetic and Natural Functional

Molecule Chemistry of Ministry of Education,

The College of Chemistry and Materials Science,

Northwest University,

Xi'an 710069, China
16-DOXYL stearic acid methyl ester spin labels were applied to explore the interior of the lipid bilayer. Only the 16-DOXYL spin probe detected evident structural changes inside the lipid system due to porphyrin intercalation. Fluidity of the lipid system and the type of the porphyrin complex introduced significantly affected the intermolecular interactions, which in certain cases may result in self-assembly of metalloporphyrin molecules within the liposome membrane, reflected in the presence of new lines in the relevant ESR spectra. The most pronounced timerelated effects were demonstrated by the EYL liposomes (liquid-crystalline phase) when doped with $\mathrm{Mg}$ and $\mathrm{Co}$ porphyrins, whereas practically no spectral changes were revealed for the metal-free base and both the $\mathrm{Ni}$ and $\mathrm{Zn}$ dopants. ESR spectra of the porphyrin-doped gel phase of DPPC liposomes did not show any extra lines; however, they indicated the formation of a more rigid lipid medium. Electronic configuration of the porphyrin's metal center appeared crucial to the degree of molecular reorganization within the phospholipid bilayer system.

Keywords Liposomes - Lipid bilayer ·

Metalloporphyrins · Electron spin resonance .

Density functional theory calculations

\section{Introduction}

Liposomes have been widely applied in medical treatment as intelligent drug carriers and this natural ability is expected to bring about a breakthrough particularly in cancer therapy [1]. The development of new curing methods involving visible and/or soft UV radiation (photodynamic therapy) gave rise to an extensive search for more or less sophisticated components which would adapt 
well to the biochemical conditions within cell membranes. Good candidates seem to be macrocyclic organometallic compounds, e.g., diverse porphyrin analogues (Figs. 1,2). The great interest in liposome-porphyrin systems results from the finding that some metalloporphyrins is as well as other macrocyclic analogues exhibit some kind of affinity for tumor cells. Namely, they possess a peculiar feature of accumulating in tumors, thus providing a way to localize and even eliminate malignant tissues in a bed of normal
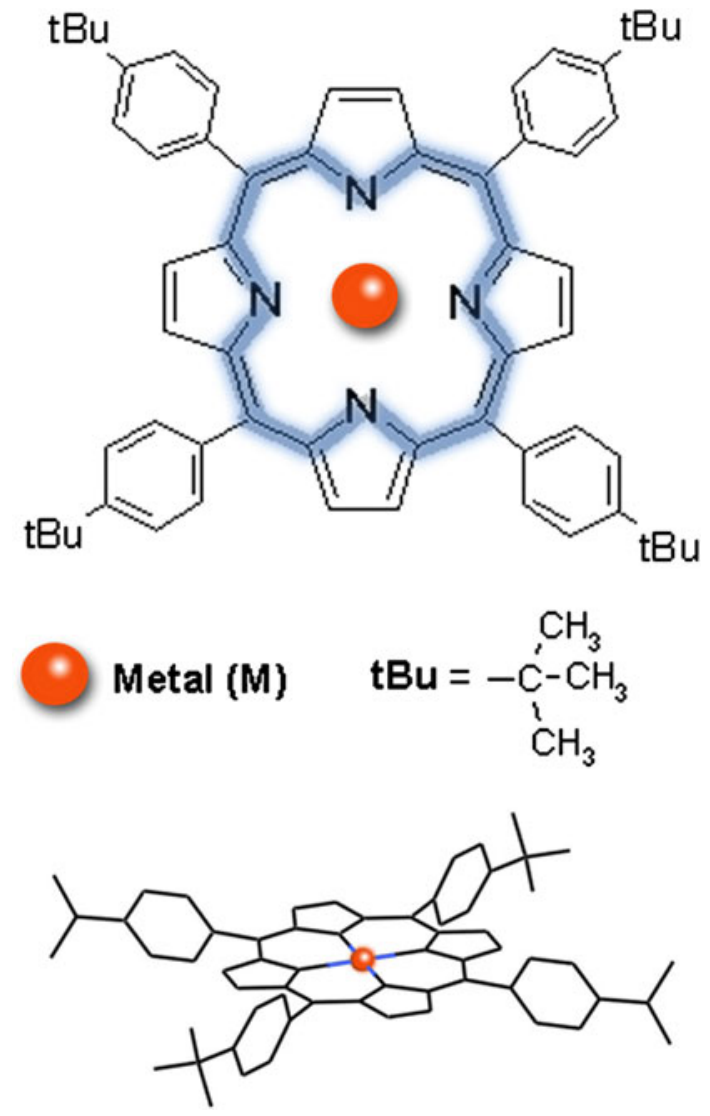

Fig. 1 General chemical structure and wire-frame representation of the metalloporphyrin molecule; the $\pi$-electronic core of the porphyrin moiety has been highlighted cells [2]. Additionally, porphyrins and similar compounds (e.g., phthalocyanines) were found to combine $\mathrm{O}_{2}$ into more or less stable adducts, which may act as effective synthetic oxygen carriers [3-5]. A succinct characterization of the porphyrin family and related molecular systems may be found in a number of publications [6-9].

The significance of these unique molecules follows first from their biochemical activity. Porphyrin complexes of iron (heme) and magnesium (chlorophylls) have been rated among the most important species found in natural biological systems. Synthetic porphyrins to some extent resemble the natural ones. Moreover, one may largely design their chemical structure to tailor their individual properties to meet specific application needs [10]. For instance, functionalization of the porphyrin moiety at the meso position, such as shown in Fig. 1, would enhance the solubility of the compound in a lipid ambient. The central part of a liposome membrane may therefore appear to be a friendly medium for strongly hydrophobic and at the same time lipophilic porphyrins. Hence, porphyrin-doped liposome structures may become interesting alternatives for versatile medical applications. However, their commercial use would first involve detailed knowledge, in particular, of the time-related physicochemical behavior of lipid membranes combined with such biologically active macrocyclic species.

The stability of a liposome structure depends on the fluidity of the lipid bilayer, which may change under the influence of various factors. Organometallic compounds when embedded into lipid membranes were reported to alter their physical properties [11-13]. Changes in the concentration of the doping agent within the membrane [14-17] as well as the ambient temperature were found to affect the fluidity of the lipid double layer [18, 19]. In previous work, the development of fluidity changes in modified membranes revealed diverse kinetics, thus showing a significant time dependence, as proved in the case of liposome doping by organic tin compounds [20] and metalloporphyrins [21]. These results unambiguously (a)
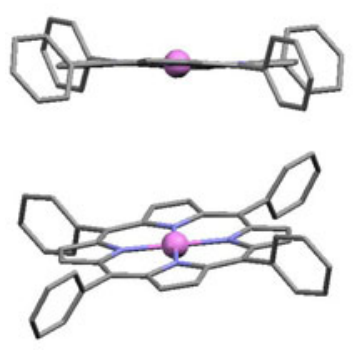

(b)
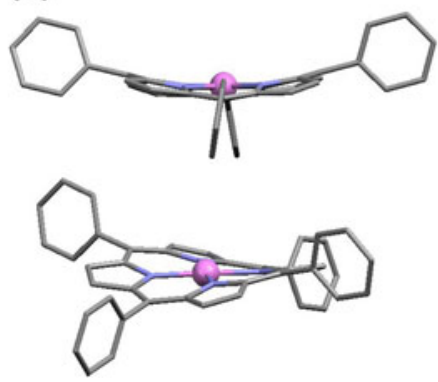

(c)

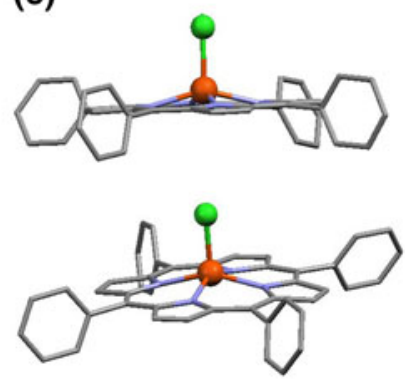

Fig. 2 Basic stereochemical features of typical four- and fivecoordinated metalloporphyrins, manifesting the flexibility of the porphyrin moiety: a flat $\operatorname{CoPp}\left(\mathrm{D}_{4 \mathrm{~h}}\right)$, b ruffled $\operatorname{CoPp}\left(\mathrm{D}_{4 \mathrm{~h}}\right)$, c axially ligated FeClPp $\left(\mathrm{C}_{4 \mathrm{v}}\right)$, where $\mathrm{Pp}$ is $\mathrm{C}_{60} \mathrm{H}_{60} \mathrm{~N}_{4}$, the porphyrin ligand. All structures are adapted from the Cambridge Structural Database (CSD) [26, 27]: TEFPID, TPORCP12, and KANYUT, respectively 
indicated the existence of interactions between the molecules of the dopant and the lipids making up the membrane. Since the lipid double layer is a dynamic formation of particles in constant movement, the admixture distribution is certainly a complex process apparently related to the membrane's fluidity and individual properties of the dopant.

From structural considerations it follows that for magnesium and the first-row transition metals the relevant metalloporphyrin molecules of the sort depicted in Fig. 1 (the same as studied in this work) are approximately of the same size and would fit well into a frame of $1.5 \times 1.7 \mathrm{~nm}^{2}$ [22]. On the basis of the dimensions of the bilayer setup reported elsewhere [23-25], one may assume there should be enough space between the lipids making up the bilayer to accommodate the porphyrins as well as to allow them to penetrate the center of the membrane. Nevertheless, the organization of the dopant molecules within the membrane could depend also on individual stereochemical features, as displayed in Fig. 2. Obviously, such structural diversity results from the electron density distribution within the bonding system of the porphyrin complex and external medium-related factors, which might have affected it. Interestingly, a number of metalloporphyrins of the same type may adopt each of these structures and they do not show any particular metal-related selectivity [26, 27].

The need for information about possible functional consequences of interactions within a doped lipid bilayer prompted us to investigate the changes that occur in porphyrin-modified liposome membranes both in the liquidcrystalline phase and in the gel phase as a function of time. Electron spin resonance (ESR) techniques were shown to be a valuable tool to explore the liposome medium and hence have been widely used to study diverse biological membrane systems particularly under physiological conditions [28, 29]. The ESR method using the 5-DOXYL, 12DOXYL, and 16-DOXYL spin labels was chosen because this set of probes would allow penetration of a large part of the membrane's interior [30], in particular the central region of the lipid bilayer where the membrane-embedded metalloporphyrin molecules were expected to be dispersed [21].

\section{Materials and methods}

Liposome preparation and measurement technique

Liquid-crystalline liposomes (main phase transition temperature $-5^{\circ} \mathrm{C}$ ) were prepared from egg yolk lecithin (EYL), whereas the liposomes with the gel-phase lipid bilayer (main phase transition temperature $41^{\circ} \mathrm{C}$ ) were obtained from dipalmitoylphosphatidylcholine (DPPC) synthetic lecithin. EYL was extracted in the laboratory of the Faculty of Chemistry (Opole University) and DPPC was purchased from Fluka. The liposomes were formed in distilled water in a sonication process by means of an ultrasonic disintegrator. For a single sample of $2 \mathrm{ml}$, the total effective sonication time was $8 \mathrm{~min}$ in alternate cycles including $30 \mathrm{~s}$ of sonication and $60 \mathrm{~s}$ of cooling. The concentration of both EYL and DPPC in the particular samples was $0.04 \mathrm{M}$ and that of the spin label was $0.5 \mathrm{~mol} \%$ (molar ratio to lipid of 0.005 ).

The spin labels 5-DOXYL, 12-DOXYL, and 16DOXYL stearic acid methyl esters (Sigma-Aldrich) were used: 2-(4-methoxy-4-oxobutyl)-4,4-dimethyl-2-tridecyl-3oxazolidinyloxy (5-DOXYL), 2-hexyl-2-(11-methoxy-11oxoundecyl)-4,4-dimethyl-3-oxazolidinyloxy (12-DOXYL), and 2-ethyl-2-(15-methoxy-15-oxopentadecyl)-4,4-dimethyl-3-oxazolidinyloxy (16-DOXYL). They were added to the particular samples during the sonication process. Porphyrins (dissolved in chloroform) were fed into a chloroform solution of the relevant lecithin. After evaporation of the solvent, water was added and the liposomes were formed. The optimum amount of the dopants was set experimentally and it was fixed at 3\% (molar) relative to the EYL and/or DPPC quantity. At this concentration, the liposome membrane system has been presumably "saturated" by the porphyrin admixture, because higher doses of the metalloporphyrin component affected neither the relaxation rate of the spin label nor the shape of the ESR spectra.

An MX-201R ESR spectrometer (TU Wrocław, Poland) was used in the tests under the following working conditions: modulation amplitude $0.032 \mathrm{mT}$, microwave power 600-620 $\mathrm{mW}$ (damping $1 \mathrm{~dB}$ ). Investigations were carried out at a constant temperature of $24^{\circ} \mathrm{C}$ using the ESR spin probe technique and the samples were placed in special glass tubes $(1 \mathrm{~mm}$ in diameter, approximately $0.04 \mathrm{~cm}^{3}$ sample volume). The spectra were collected from the moment the additive had been fed in and then for the following $350 \mathrm{~h}$. Between measurements, the samples were stored in the dark at $24^{\circ} \mathrm{C}$ in a sealed container. Air contact took place only during the measurement itself.

In all experiments, the scanning range and the sweep speed of the ESR spectrometer were $5 \mathrm{mT}(50 \mathrm{G})$ and $256 \mathrm{~s}$, respectively, and the results represent an average of three to five tests for an individual sample.

From the ESR spectrum (Fig. 3) the spectroscopic parameter $\tau$ (rotational correlation time) may be estimated. For the systems studied, the values of $\tau$ were primarily determined and reported in a previous work [21]. The parameter's value depends, among other factors, on the degree of the membrane's fluidity and is greater the more rigid (better ordered) the medium is in which the "doxyl" radical of the spin probe has been situated [29-32]. 
$\tau=5.95 \cdot \Delta H_{0} \cdot\left(\sqrt{\frac{I_{o}}{I_{+1}}}+\sqrt{\frac{I_{0}}{I_{-1}}}-2\right) \cdot 10^{-10} \mathrm{~s}$

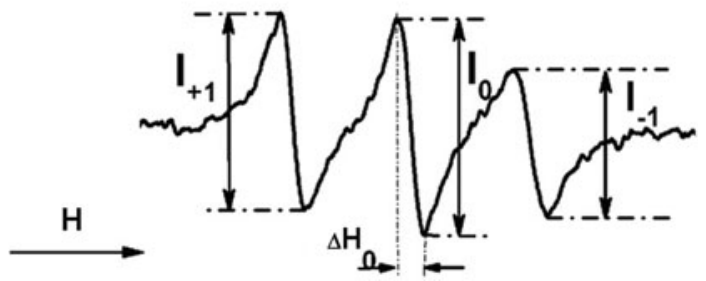

Fig. 3 Electron spin resonance (ESR) spectrum of the liposomeembedded 16-DOXYL spin label. The spectroscopic parameter $\tau$ (rotational correlation time) determines the fluidity of the membrane medium penetrated by the spin probe

ESR spectra (where applicable) were analyzed using a deconvolution procedure provided by the OriginLab 8.0 software package.

Characterization of the porphyrin material

Metalloporphyrins (MPp, where $\mathrm{M}$ is a metal ion or two hydrogen atoms and $\mathrm{Pp}$ is $\mathrm{C}_{60} \mathrm{H}_{60} \mathrm{~N}_{4}$, the porphyrin ligand) including $\mathrm{Mg}(\mathrm{II}), \mathrm{Mn}(\mathrm{III}), \mathrm{Fe}(\mathrm{III}), \mathrm{Co}(\mathrm{II}), \mathrm{Ni}(\mathrm{II}), \mathrm{Cu}(\mathrm{II})$, and $\mathrm{Zn}$ (II) ions as well as the metal-free base $\left(\mathrm{H}_{2} \mathrm{Pp}\right.$, merely for comparison) were used as the dopants. The Pp ligand is a 5,10,15,20-tetrakis(4-tert-butylphenyl)porphyrin. General and molecular structures of the compounds are shown in Fig. 1. The coordination symmetry was square planar $\left(\mathrm{D}_{4 \mathrm{~h}}\right)$ in the case of $\mathrm{Mg}(\mathrm{II}), \mathrm{Co}(\mathrm{II}), \mathrm{Ni}(\mathrm{II}), \mathrm{Cu}(\mathrm{II})$, and $\mathrm{Zn}(\mathrm{II})$ complexes (Figs. 1, 2a) and rectangular $\left(\mathrm{D}_{2 \mathrm{~h}}\right)$ for $\mathrm{H}_{2} \mathrm{Pp}$. However, $\mathrm{Mn}(\mathrm{III})$ and $\mathrm{Fe}(\mathrm{III})$ porphyrins represent a five-coordinated complex type $\left(\mathrm{C}_{4 \mathrm{v}}\right.$ symmetry, Fig. 2c) with one axial ligand attached to the metal, $\mathrm{CH}_{3} \mathrm{COO}^{-}$and $\mathrm{Cl}^{-}$, respectively. All porphyrins used in this work were synthesized according to the method described in a previous paper and were characterized in detail therein [33].

\section{Theoretical studies}

To support the explanation of the ESR results, unrestricted density functional theory calculations were performed to establish the most probable spin state for the porphyrincoordinated $\mathrm{Mn}(\mathrm{III}), \mathrm{Fe}(\mathrm{III}), \mathrm{Co}(\mathrm{II})$, and $\mathrm{Ni}(\mathrm{II})$ ions. For this reason, a porphine moiety model (Fig. 4) was used, i.e., a simplified metalloporphyrin structure, as reported elsewhere [34-37]. The principal objective was to estimate the relative energy $(\Delta E)$ of the compounds versus the electron density distribution within the coordination sphere, for any possible electronic configuration of the central metal ion. Detailed results are presented in the electronic supplementary material; for the $\Delta E$ values
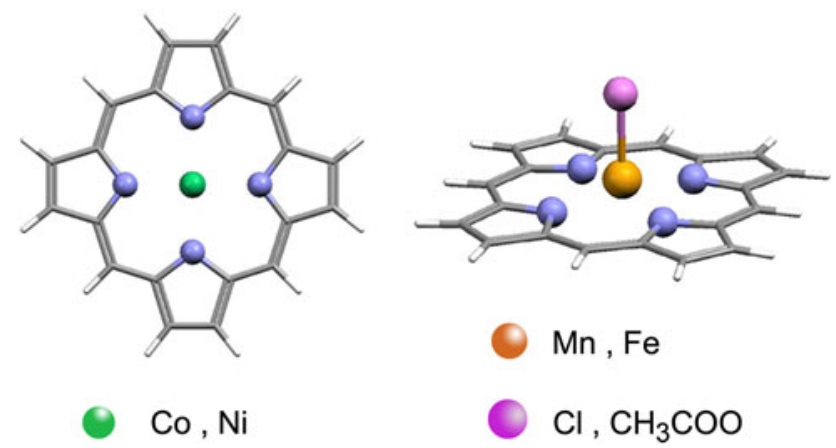

Fig. 4 The simplified molecular structures considered for density functional theory calculations

determined and the optimized structures, see Table S1 and Fig. S7, respectively.

The computation procedure involved the Becke threeparameter exchange functional [38] with the Lee-YangParr correlation functional [39] and the meta-generalized gradient approximation functional with Tao-Perdew-Staroverov-Scuseria (TPSS) exchange and TPSS correlation [40] along with the LANL2DZ basis set [41]. Geometry optimization and vibrational analysis were performed without constraints on isolated molecules with the Gausssian 03 package [42].

\section{Results and discussion}

Two diverse lipid systems, EYL and DPPC, of different chemical and physical properties were intentionally chosen to show how far an intercalation by relatively bulky lipophilic porphyrins (Fig. 1) can modify the membrane's initial structure in the course of time. It must be remembered, however, that natural membranes are much more like those of EYL liposomes and hence in this study DPPC was considered rather in terms of a reference model system. Furthermore, during the 350-h period of testing, the undoped liposomes and also some of the intercalated EYL and DPPC membrane systems practically did not change chemically, as follows from the ESR spectra reported in Figs. S1-S4. Such an observation means that the liposomes mentioned did not suffer any significant peroxidation of the lipid chains, which might have affected the experimental results. Therefore, this general conclusion may equally be applied to the other MPp-doped liposome systems studied described in detail later.

\section{The EYL lipid system}

The diverse spin probes used in the study, owing to their specific molecular structure, may sense alterations in the membrane's medium condition at different levels across 

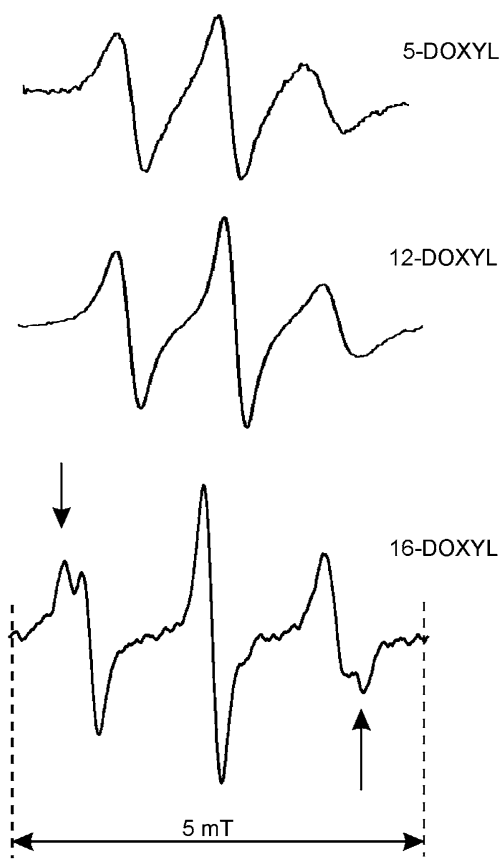

Fig. 5 ESR spectra displayed by diverse DOXYL spin labels sensing different levels inside of the lipid bilayer of egg yolk lecithin (EYL) liposomes doped by $\mathrm{MnPp}$ (measured after $350 \mathrm{~h}$ of testing) featuring the development of extra spectral components due to a dopant-driven effect localized in the membrane's center

the phospholipid bilayer; in the upper chain and headgroup region (5-DOXYL), in the middle chain region (12DOXYL), and in the lower lipid chain region, i.e., membrane's center (16-DOXYL) [30]. A comparison of the results shown in Fig. 5, referred to a selected liposome system, indicates that the porphyrins have penetrated deeply into the membrane's interior owing to hydrophobic interactions with the lipid chains (see also the extensive data in the supplementary electronic material). This conclusion follows directly from the characteristic splitting of the low-field and high-field resonance lines, detected exclusively by the 16-DOXYL spin label. Hence, one may have assume the most meaningful changes induced within the membrane due to doping occur rather in the central part of the bilayer. For this reason, further discussion is based predominantly on the data provided by the 16-DOXYL probe; however, the spectra regarding the 5-DOXYL and 12-DOXYL spin labels are shown and are discussed in the electronic supplementary material (Figs. S3, S4).

Representative spectra of the 16-DOXYL spin probe illustrating the consequence of EYL liposome doping are presented in Fig. 6. The membranes show the liquid-crystalline phase. The Spectra shown on the left refer to the initial stage of the experiment, immediately after the admixtures were fed into the liposomes, whereas those on the right apply to its final phase, i.e., $350 \mathrm{~h}$ after the liposomes were formed.

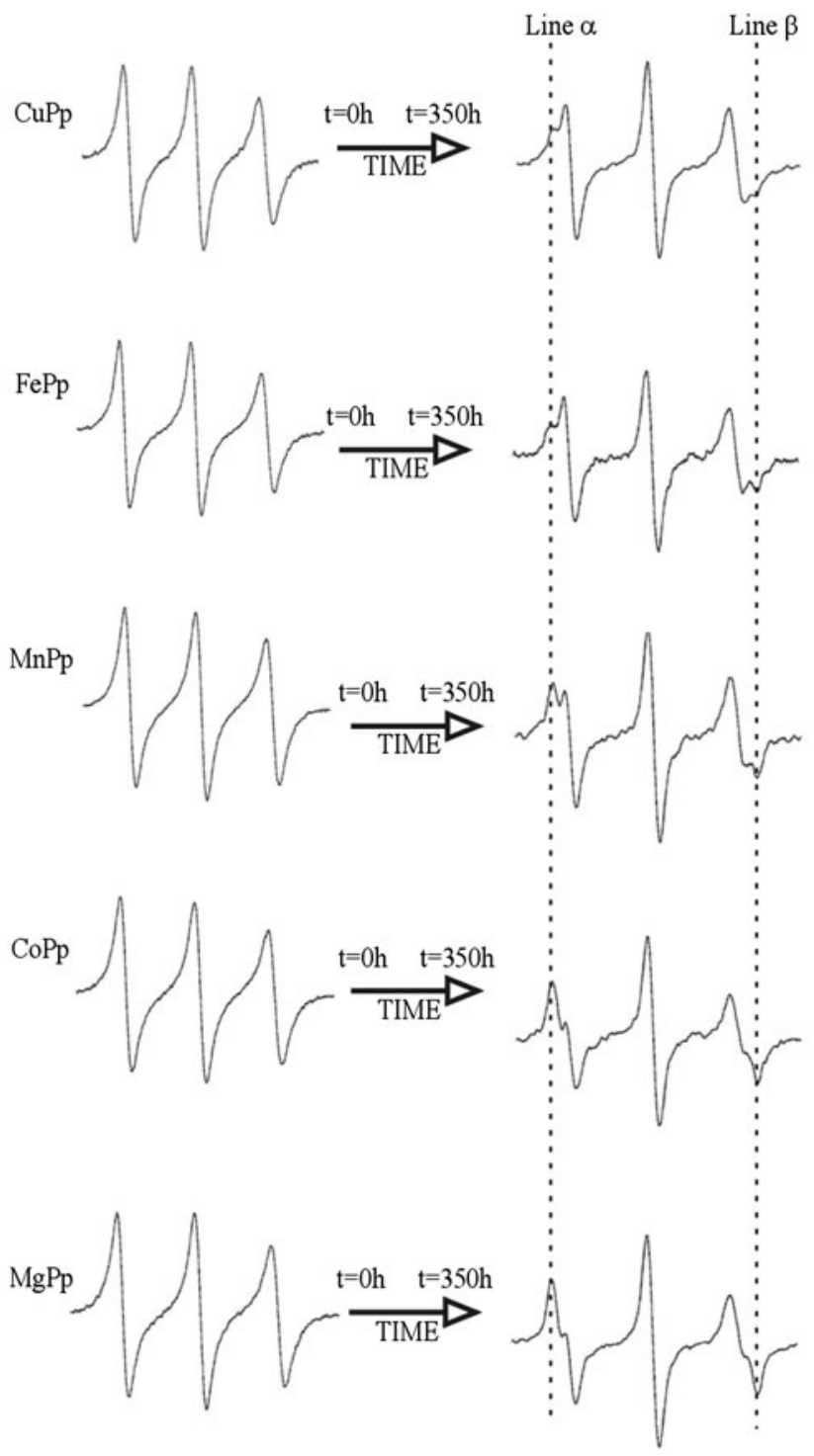

Fig. 6 ESR spectra of the 16-DOXYL spin label embedded inside of the EYL lipid bilayer including admixtures of CuPp, FePp, MnPp, $\mathrm{CoPp}$, and $\mathrm{MgPp}$, registered at the initial and final stages of the experiment (scanning range $5 \mathrm{mT}$ ); the intensity of the newly emerged $\alpha$ and $\beta$ lines depends on the dopant

As follows from Fig. 6, initially all samples display a typical motional-averaged three-line ESR spectrum. In the course of time, the character of the spectra evidently changed for liposomes doped with $\mathrm{MgPp}, \mathrm{MnPp}, \mathrm{FePp}$, $\mathrm{CoPp}$, and CuPp. Two new lines appeared outside the original spectrum, line $\alpha$ at lower and line $\beta$ at higher magnetic field. This was not seen, however, in the spectra registered for the reference liposome sample (undoped) as well as for those including $\mathrm{H}_{2} \mathrm{Pp}$, NiPp, and $\mathrm{ZnPp}$; none of these displayed any new spectral lines during the whole experiment (Fig. S1). The most pronounced changes are in the ESR spectra of EYL liposomes doped with MgPp and CoPp, which both show strong $\alpha$ and $\beta$ lines, whereas in the 
case of MnPp and particularly for FePp and CuPp, although evident, these lines are considerably less intense (Fig. 6). They appeared first in the spectra of liposomes containing $\mathrm{MgPp}$ and CoPp (after $250 \mathrm{~h}$ ) and latest for those doped with FePp and CuPp (after $300 \mathrm{~h}$ ). The arrangement of the ESR spectra in Fig. 6 allows one to track the increase in intensity of $\alpha$ and $\beta$ lines from the initial stage (admixture of CuPp) through the intermediate stage (admixture of $\mathrm{MnPp}$ ) to the final stage (admixture of MgPp). Note that in the latter case, almost the entire ESR signal recorded originates from the new resonance lines, which appeared in the spectrum. Such results suggest that MgPp and CoPp display considerably higher activity in the membrane's lipid system than do FePp and CuPp. In a previous paper [21] we reported FePp and CuPp definitely show the least potential in fluidizing the inside of the lipid double layer of EYL liposome membranes, and this finding matches well with the results obtained in the present work.

To identify the possible origin of the observed splitting, a theoretical approximation of the experimental ESR data was performed using a deconvolution procedure, which allowed the extraction of two individual components from the recorded spectra, as shown in Fig. 7. These were used to generate the theoretical ESR spectrum. Since the simulated spectra almost perfectly fitted the experimental ones, it became evident that the spectra reported on the right in Fig. 6 demonstrate a set of two superimposed three-line spectra typical for the fast rotating 16-DOXYL spin label. A divergent shift of the new lines in the ESR spectrum featuring some higher value of the ESR coupling coefficient indicates a change in polarity of the immediate surroundings of the spin label, e.g., due to a local increase in electron density [28]. This would imply the MPp-induced

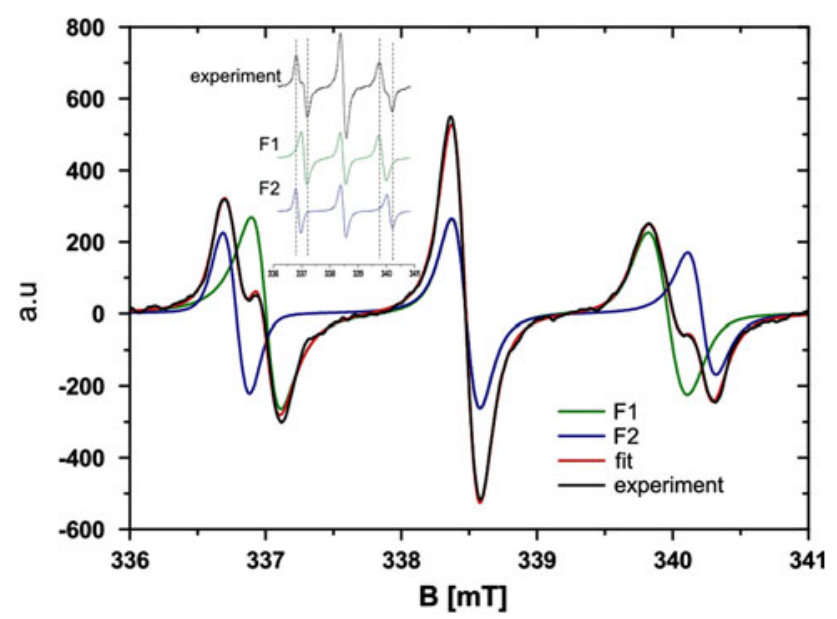

Fig. 7 Deconvolution analysis of the 16-DOXYL ESR spectrum recorded after $350 \mathrm{~h}$ for EYL liposomes doped by CoPp (experiment) and the resultant simulated spectrum (fit) involving the sum of components F1 and F2; the inset shows the experimental spectrum and its two independent components formation of a new phase within the membrane studied, characterized by enhanced electron density compared with the lipid phase alone. Thus, one ESR component (F1) reflects the rotation of the spin label embedded in a highordered lipid environment, whereas the other one (F2) reflects the lipid structure distorted by the effect of the porphyrin dopant.

\section{The DPPC lipid system}

Unlike for EYL, in case of the liposomes produced from DPPC lecithin the membranes were in the gel phase. In fact, one could notice thickening of the DPPC liposomes shortly after doping, by watching the samples becoming more and more turbid; this was not manifested by the EYL liposomes. Therefore, after some preliminary attempts involving the diverse spin markers, only the 16-DOXYL spin label was chosen for experiments dedicated to the DPPC membrane. In principle, the measured ESR spectra revealed only the aforementioned decrease in fluidity. Even though in the course of time we observed some slight changes in the ESR spectra of the MPp-doped DPPC liposomes, none of them displayed extra lines (Fig. S2). The value of $\tau$ (rotational correlation time) determined for the spin probe localized within DPPC liposome membranes was $25.8 \times 10^{-10} \mathrm{~s}$, whereas in the EYL case it was $4.2 \times 10^{-10} \mathrm{~s}$, which means the rotational motion of the spin probe was many times faster within the EYL liposome membranes than for the DPPC ones. This implies the essential condition to generate a new phase is the liquidcrystalline state of the membrane in which metalloporphyrin species have been dispersed. Presumably, only the liquid-crystalline phase allows large MPp molecules embedded within the membrane's lipid medium to achieve the necessary mobility to develop in the course of time some new structures characterized by enhanced polarity. However, in the definitely more rigid gel phase of DPPC liposomes, the porphyrins (after sonication) once fed into the membrane seem to remain "immobilized" among the lipid tails, showing a bilayer structure like that of a "raisinfilled cake." Therefore, no new lines could be observed in the related ESR spectra.

\section{Metal spin state and the ESR spectra}

Some of the porphyrins used feature a central metalderived nonzero total spin ( $\mathrm{Mn}, \mathrm{Fe}, \mathrm{Co}$, and $\mathrm{Cu}$ ) and hence one should consider the possibility of spin-spin interaction with the ESR spin label. However, from the recorded spectra it follows that this might not be the case. Note that the porphyrins including paramagnetic ions produce a similar trend in spectral changes, as does the diamagnetic MgPp dopant, which in turn was not revealed by the other 
diamagnetic NiPp or ZnPp species (Figs. 6, S1-S4). Besides, the very small concentration of the dopants would preclude the revelation of any evidence of the metal spin sensing by the 16-DOXYL spin probe. None of the porphyrins tested displayed ESR signals even when dispersed in the unlabeled EYL membranes, presumably owing to limited solubility of the porphyrins in the liposome material. Therefore, in the further discussion regarding the phenomenon observed in the spectra displayed on the right in Fig. 6, we have assumed the above-mentioned spin-spin interactions do not contribute to the observed effects. Additional related material has been included in the electronic supplementary material (Fig. S5).

Distribution of metalloporphyrins within the EYL membrane

Undoubtedly, the metalloporphyrins play an important role in generating the new polar phase within the lipid bilayer. The final effect they produced depended on the kind of complexed metal and this relation was reflected clearly in the ESR spectra reported in Fig. 6. The lipophilic porphyrins used in this study diffuse into the membrane's interior relatively easily and initially seem to be rather evenly distributed within it. In the course of time, owing to interactions with the flexible lipid's tales and other forces related to the macrocycle's bonding system, the separate metalloporphyrin molecules may be driven to assemble and organize into specific domains, characterized by enhanced electron density (Fig. 8). The porphyrins owe such behavior to the paramagnetic properties of the incorporated metal ion. Obviously, the porphyrins would find the most favorable conditions to form some kind of molecular clusters in the center of the bilayer. Consequently, the original arrangement of lipids within the membrane will be more or less disrupted. Thus, the response of spin labels located nearby is reflected by the appearance of a new ESR spectrum, as discussed earlier (Figs. 6, 7).

Aggregation of porphyrins is commonly known and observed in diverse systems. It has been generally attributed to interactions of various kinds between the $\pi$ electron setups of adjacent macrocycles, including noncovalent bonding effects, reflecting the specific chemical nature of metalloporphyrins [43]. Thus, under favorable conditions, such interactions may eventually generate more or less desirable new liposome-porphyrin structures [44].

In a hydrophobic lipid medium, the "face-to-face" interactions involving meso-substituted MPp molecules may prove more or less effective, unless these molecules are prevented from getting closer by either steric hindrance or electron repulsions, or both. The electron density distribution in the vicinity of the metal core might be crucial to this point, and the actual spin state may not correspond to the intuitively and/or experimentally (ESR) predicted one. In fact, it depends on the properties of the surrounding medium $[45,46]$, the presence of $\mathrm{O}_{2}[47,48]$, axial ligands [35], and functional groups [49], as well as configuration effects revealed, e.g., by the "ESR-silent" Mn(III) porphyrins [50]. In fact, under the liposome-dedicated measurement conditions, we obtained reliable ESR signals only for $\mathrm{CuPp}$ and $\mathrm{CoPp}$ (concentrated solutions in $\mathrm{CH}_{2} \mathrm{Cl}_{2}$ ) as presented in Fig. S5.

From the analysis of spin population it follows that in $\mathrm{Co}$ (II) and $\mathrm{Ni}$ (II) the low-spin state is favored, whereas $\mathrm{Mn}(\mathrm{III})$ and $\mathrm{Fe}(\mathrm{III})$ prefer higher spin states (Table S1), which generally fits the literature data. However, a low $\Delta E$ (close to 0 ) value may indicate the possibility of mixed spin populations (e.g., for $\mathrm{Fe}(\mathrm{III})$ [34, 49]) and thus diverse ESR results (e.g., for $\mathrm{Co}(\mathrm{II})$, [45-48]). The nonpolar lipid environment seems to favor the states corresponding to $\Delta E=0$ (except for $\mathrm{Fe}^{\mathrm{III}} \mathrm{Pp}$ ), which was confirmed in the trend demonstrated in Fig. 6, as discussed below.

On the basis of the theory of the square-planar ligand field, one can assume that for the first-row transition metal porphyrins the metal's $d_{z^{2}}$ orbital (perpendicular to the macrocycle plane) is at the outmost only weakly engaged in bonding with the macrocycle $[34,51]$ (see also Fig. S6). Considering the preferred electronic configurations (Table S1, Fig. S6), one can assume that $\mathrm{NiPp}$ and $\mathrm{ZnPp}$ may not be susceptible to face-to-face interactions owing to the
Fig. 8 Hypothetical simulation of metalloporphyrin migration inside a liquid-crystalline liposome membrane, leading up to the creation of molecular clusters and disorder of the lipid setup

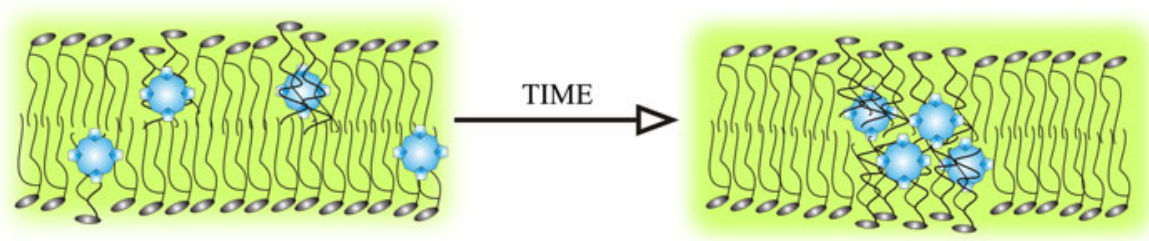

Metalloporphyrin molecule

Lipid molecule 
filled $d_{z^{2}}$ orbital. In $\mathrm{Cu}$ (II) the $d_{z^{2}}$ orbital is also filled; however the $d_{x^{2}-y^{2}}$ orbital is only half occupied, which presumably accounts for weak attractive interactions (interporphyrin) between CuPp species. Axial ligation of the $\mathrm{Fe}(\mathrm{III})$ and $\mathrm{Mn}$ (III) ions might have reduced the association capacity of $\mathrm{FePp}$ and MnPp. Some enhanced activity observed in the latter case may be assigned to the higher ground-state total spin found for the Mn(III) $d^{4}$ ion. The $d^{7}$ low-spin $\operatorname{Co}\left(\right.$ II) with a half-filled $d_{z^{2}}$ orbital may be expected to promote the self-assembly of CoPp species. $\mathrm{Mg}$ (II) is a closed-shell ion with the electron density distributed inside the porphyrin core, thus allowing more effective "face-to-face" interactions between adjacent $\mathrm{MgPp}$ molecules, compared with the other porphyrins studied. The behavior of the metal-free $\mathrm{H}_{2} \mathrm{Pp}$ could be assigned either to intermolecular repulsions caused by the two inner hydrogens evidently sticking out of the porphyrin core or to possible bonding-like interactions between $\mathrm{H}_{2} \mathrm{Pp}$ and the lipids, mediated by the hydrogen atoms already mentioned [52]. Thus, the spin-state-related self-assembly trend in the order $\mathrm{H}_{2} \mathrm{Pp} \approx \mathrm{ZnPp} \approx \mathrm{NiPp}<\mathrm{CuPp} \approx$ $\mathrm{FePp}<\mathrm{MnPp} \ll \mathrm{CoPp}<\mathrm{MgPp}$ has been perfectly reflected in the ESR spectra shown in Fig. 6. Modifications of liposomal structures similar to those suggested due to porphyrin intercalation have found support in our recent theoretical study based on a computer-simulated model membrane [53].

Summarizing the reported results, we wish to highlight the principal reasons why in a lipid bilayer its initial wellorganized setup may be considerably rearranged by species able to penetrate it. The resulting arrangement of membrane components is a compromise between the stereochemical properties (steric hindrance, molecular structure flexibility [44, 54]) and electrostatic interactions resulting from distribution of electron density in the metal's coordination sphere. Therefore, in the case of liquid-crystalline liposomes some of the metalloporphyrins once embedded into the membrane show a peculiar susceptibility to selfassemble into larger molecular structures. The relevance of this finding follows from the fact that any disruption within the well-organized lipid structure of a cell membrane may considerably affect the vital function of the cell itself. Controlled membrane modification leading to its presumed dysfunction may be interesting for medical reasons and of particular significance in tumor treatment, especially as diverse porphyrins and their derivatives have been shown to accumulate selectively in cancer cells [2]. The porphyrins owe this peculiar feature principally to their ability to permeate through the phospholipid bilayer and to dislocate within the membrane, as confirmed in our work. At this point, ESR studies appear very useful in providing important data concerning molecular organization in cell membranes that should help assess the actual condition and functionality of the relevant liposomes.

Obviously, there are still a number of unsolved important problems, such as the orientation and ordering of the macrocycles within the lipid medium, the influence of the postulated porphyrin aggregation upon the stability of the bilayer, and the membrane's strength and its surface properties, as well as the functional consequences of liposome doping. These topics merit detailed examination and are considered a key issue to be explored in our currently realized liposome research projects.

Acknowledgments Theoretical density functional theory calculations were supported by a grant obtained from the Academic Computer Center CYFRONET AGH Kraków, no. KBN/SGI2800/ UOpolski/012/2001.

Open Access This article is distributed under the terms of the Creative Commons Attribution Noncommercial License which permits any noncommercial use, distribution, and reproduction in any medium, provided the original author(s) and source are credited.

\section{References}

1. Immordino ML, Brusa P, Arpicco S, Stella B, Dosio F, Cattel L (2003) J Control Release 91:417-429

2. Bonnett R (1989) New Sci 55-58

3. Basolo F, Hoffman BM, Ibers JA (1975) Acc Chem Res 8:384-392

4. Wechsler CJ, Hoffman BM, Basolo F (1975) J Am Chem Soc 97:5278-5280

5. Słota R, Dyrda G, Hnatejko Z, Karolczak J, Stryła Z (2006) J Porphyr Phthalocyanines 10:43-54

6. Fleischer EB (1970) Acc Chem Res 3:105-112

7. Kai E, Nishimoto K (1982) Bull Chem Soc Jpn 55:1242-1245

8. Marsh DF, Mink LM (1996) J Chem Educ 73:1188-1190

9. D'Souza F, Boulas P, Aukauloo AM, Guilard R, Kisters M, Vogel E, Kadish KM (1994) J Phys Chem 98:11885-11891

10. Wang C, Yang G, Li J, Mele G, Słota R, Broda MA, Duan M, Vasapollo G, Zhang X, Zhang FX (2009) Dyes Pigm 80:321-328

11. Falcioni G, Gabiannelli R, Santini A, Zolese G, Griffits D, Bertoli E (1996) Appl Organomet Chem 10:451-457

12. Gray BH, Porvaznik M, Flemming C, Lee LH (1987) Cell Biol Toxicol 3:23-38

13. Kleszczyńska H, Sarapuk J, Przestalski S (1999) Folia Histochem Cytobiol 37:1-31

14. Podolak M, Man D (2002) Cell Mol Biol Lett 7:961-969

15. Podolak M, Engel G, Man D (2006) Z Naturforsch C 61:453-457

16. Man D, Podolak M, Engel G (2006) Cell Mol Biol Lett 11:56-61

17. Man D, Podolak M (2007) Z Naturforsch C 62:427-432

18. Podolak M, Man D, Przestalski S (1998) Z Naturforsch C 53:191-196

19. Enders A, Nimtz G (1984) Ber Bunsenges Phys Chem $88: 512-517$

20. Man D (2008) J Liposome Res 18:225-234

21. Man D, Słota R, Mele G, Li J (2008) Z Naturforsch C 63:440-444

22. Słota R, Mele G, Ejsmont K, Domański AA, Del Sole R (2007) Acta Crystallogr E 63:m2582 
23. Möhwald H (1990) Annu Rev Phys Chem 41:441-476

24. Stigter D, Mingins J, Dill KA (1992) Biophys J 61:1616-1629

25. Kubica K (2002) Comput Chem 26:351-356

26. Allen FH (2002) Acta Crystallogr B 58:380-388

27. Orpen AG (2002) Acta Crystallogr B 58:398-406

28. Harrison R, Lunt GG (1975) Biological membranes. Their structure and function. Blackie, Glasgow

29. Hemminga MA (1983) Chem Phys Lipids 32:323-383

30. Vogel A, Scheidt HA, Huster D (2003) Biophys J 85:1691-1701

31. Cannon B, Polnaszek CF, Butler KW, Eriksson LE, Smith ICP (1975) Arch Biochem Biophys 167:505-518

32. Schreier S, Polnaszek CF, Smith ICP (1978) Biochim Biophys Acta 515:395-436

33. Mele G, Del Sole R, Vasapollo G, García-López E, Palmisano L, Li J, Słota R, Dyrda G (2007) Res Chem Intermed 33:433-448

34. Cheng RJ, Chen PY, Lovell T, Liu T, Noodleman L, Case DA (2003) J Am Chem Soc 125:6774-6783

35. Praneeth VKK, Neese F, Lehnert N (2005) Inorg Chem 44:2570-2572

36. Atanasov M, Daul CA, Rohmer M-M, Venkatachalam T (2006) Chem Phys Lett 427:449-454

37. Praneeth VKK, Paulat F, Berto TC, DeBeer George S, Näther C, Sulok CD, Lehnert N (2008) J Am Chem Soc 130:15288-15303

38. Becke AD (1993) J Chem Phys 98:1372-1377

39. Lee C, Yang W, Parr RG (1988) Phys Rev B 37:785-789

40. Tao J, Perdew JP, Staroverov VN, Scuseria GE (2003) Phys Rev Lett 91:146401

41. Hay PJ, Wadt WR (1985) J Chem Phys 82:270-283

42. Frisch MJ, Trucks GW, Schlegel HB, Scuseria GE, Robb MA, Cheeseman JR, Montgomery JA Jr, Vreven T, Kudin KN, Burant JC, Millam JM, Iyengar SS, Tomasi J, Barone V, Mennucci B, Cossi M, Scalmani G, Rega N, Petersson GA, Nakatsuji H, Hada M, Ehara M, Toyota K, Fukuda R, Hasegawa J, Ishida M, Nakajima T, Honda Y, Kitao O, Nakai H, Klene M, Li X, Knox JE, Hratchian HP, Cross JB, Adamo C, Jaramillo J, Gomperts R,
Stratmann RE, Yazyev O, Austin AJ, Cammi R, Pomelli C, Ochterski JW, Ayala PY, Morokuma K, Voth GA, Salvador P, Dannenberg JJ, Zakrzewski VG, Dapprich S, Daniels AD, Strain MC, Farkas O, Malick DK, Rabuck AD, Raghavachari K, Foresman JB, Ortiz JV, Cui Q, Baboul AG, Clifford S, Cioslowski J, Stefanov BB, Liu G, Liashenko A, Piskorz P, Komaromi I, Martin RL, Fox DJ, Keith T, Al-Laham MA, Peng CY, Nanayakkara A, Challacombe M, Gill PMW, Johnson B, Chen W, Wong MW, Gonzalez C, Pople JA (2003) Gaussian 03, revision B.04. Gaussian, Pittsburgh

43. Medaković VB, Milčič MK, Bogdanovič GA, Zarič SD (2004) J Inorg Biochem 98:1867-1873

44. Postigo F, Mora M, De Madariaga MA, Nonell S, Sagristá ML (2004) Int J Pharm 278:239-254

45. Yakubovich TN, Teslenko VV, Veisov BK, Zub YL, Parish RV (2001) Russ J Coord Chem 27:560-566

46. La Mar GN, Walker FA (1973) J Am Chem Soc 95:1790-1796

47. Bowen JH, Shokhirev NV, Raitsimring AM, Buttlaire DH, Walker FA (1997) J Phys Chem B 101:8683-8691

48. Jones RD, Summerville DA, Basolo F (1978) J Am Chem Soc 100:4416-4424

49. Ohgo Y, Neya S, Ikeue T, Takahashi M, Takeda M, Funasaki N, Nakamura M (2002) Inorg Chem 41:4627-4629

50. Goldberg DP, Telser J, Krzystek J, Montalban AG, Brunel LC, Barrett AGM, Hoffman BM (1997) J Am Chem Soc 119:8722-8723

51. Simon J, André JJ (eds) (1985) Molecular semiconductors. Springer, Berlin

52. Kano K, Fukuda K, Wakami H, Nishiyabu R, Pasternak RF (2000) J Am Chem Soc 122:7494-7502

53. Man D, Olchawa R, Kubica K (2010) J Liposome Res 20:211-218

54. Hladyszowski J, Gabrielska J, Ordon P, Przestalski S, Langner M (2002) J Membr Biol 198:213-223 